\title{
HOMOGENEOUS BUNDLES AND THE TRACE OF THE HEAT KERNEL
}

\author{
H. D. FEGAN \\ (Received 6 June 1984) \\ Communicated by K. Mackenzie
}

\begin{abstract}
We study the heat equation on a homogeneous bundle over a compact Lie group. The trace of the heat kernel is explicitly calculated. By comparing this with the formula constructed from the eigenvalues (with multiplicities) of the Laplacian we obtain an unusual formula involving the Clebsch-Gordan numbers. The main method is to use invariance under conjugation to pass from the group to its maximal torus, where a direct calculation can be carried out.
\end{abstract}

1980 Mathematics subject classification (Amer. Math. Soc.): 58 G 11.

\section{Introduction}

The link between the spectrum of differential operators on a compact manifold and the geometry of the manifold is well known. Here we content ourselves with a reference to [5]. In [5] Patodi studied the Laplacian acting on forms, that is on sections of exterior powers of the cotangent space. We note that he used the Hodge Laplacian defined by

$$
\Delta=d d^{*}+d^{*} d
$$

Our Laplacian is defined on a homogeneous bundle as in [6].

If we consider the case of the trivial bundle the work of this paper reduces to the heat equation on scalar valued functions. This has been extensively studied. The reader should note that in this case the Laplacian used here is double the usual Laplacian of functions on a Lie group.

(C) 1986 Australian Mathematical Society $0263-6115 / 86 \$ A 2.00+0.00$ 
In this paper the heat equation is studied. As is shown in [4] this is essentially the same as studying spectral invariants for the Laplacian itself. It happens that the heat equation provides a more convenient approach than studying the spectrum. However, the basic information is the same in both cases.

This paper is divided into six sections. In Section 2 is described the basic theory of the heat equation on sections of a homogeneous bundle over $G$ with the basic definitions taken from [6] and additional material from [5]. Section 3 is concerned with the case of bundles over the circle $S^{1}$. This provides both an example of the theory in Section 2 and the results of a basic calculation for later use. The next section, 4 , describes the passage between solutions on $G$ and solutions of the flat heat equation on the maximal torus $T$. Then in Section 5 we consider traces. There are several general technical lemmas. Then we put all the results together. The fundamental solution is transferred to the maximal torus. The torus is then regarded as a collection of circles and the calculation of Section 3 is used. This gives the result of Theorem 1.1.

THEOREM 1.1. The trace of the heat equation is

$$
Z(t)=\frac{e^{-8 \pi^{2}\|\rho\|^{2} t}}{|W|} \sum m_{\tau}(\alpha) d(\lambda) d(\lambda+\alpha) e^{-4 \pi^{2}\left(\|\lambda\|^{2}+\|\lambda+\alpha\|^{2}\right) t} .
$$

There is a large amount of notation in Theorem 1.1 which needs explaining. The sum is over all weights $\alpha$ and $\lambda$ of the Lie group $G, \tau$ is a dominant weight defining an irreducible representation $\pi_{\tau}$ and a homogeneous bundle $E_{\tau}$ (see Section 2 or [6] for more details). The symbol $m_{\tau}(\alpha)$ denotes the multiplicity of the weight $\alpha$ in the representation $\tau$. It is zero except for a finite number of $\alpha$ when it is a positive integer. We use \|\|$^{2}$ for the norm squared of the negative Killing form, and $\rho$ is half the sum of the positive roots. The function $d$ is the polynomial such that $d(\tau+\rho)=\operatorname{dim} \pi_{\tau}$, in the case when $\tau$ is dominant and $|W|$ is the order of the Weyl group.

In the final section, 6, we give an application of Theorem 1.1. In a previous paper [2] the Laplacian on forms was studied and a representation theoretic description of the spectrum given. The results there extend in an obvious way to any homogeneous bundle. Thus we can write down $Z(t)$ in terms of eigenvalues and their multiplicities. Comparing the result with Theorem 1.1 yields the formula

$$
\sum m_{\lambda \tau^{*}}(\mu) d(\mu+\rho)^{2}=\frac{1}{|W|} \sum m_{\tau}(\lambda-\mu) d(\lambda+\rho) d(\mu+\rho) .
$$

Here $m_{\lambda \tau^{*}}(\mu)$ are the Clebsch-Gordan numbers; in both sums $\|\lambda+\rho\|^{2}+$ $\|\mu+\rho\|^{2}=n$, a fixed constant; in the first sum $\lambda$ and $\mu$ are dominant weights, but in the second they are just weights. 
Finally we draw particular attention to one result in Section 5. The fundamental solution of the heat equation is defined as an operator in (2.8). It can be interpreted as the section

$$
H(x, t)=\sum \phi_{\lambda}(x) e^{-\lambda t},
$$

which in the case of the trivial bundle is the convolution kernel. We observe that the trace of the operator is given by

$$
\operatorname{tr} H(t)=\int\langle H(x, t), H(x, 0)\rangle d x .
$$

This leads us to define the trace of any solution, $H_{a}$, as

$$
\operatorname{tr} H_{a}(t)=\int\left\langle H_{a}(x, t), H_{a}(x, 0)\right\rangle d x
$$

(see equation (5.1)). The reason this is important to us is that in passing from the group to the torus the fundamental solution goes to a solution which is not the fundamental one. Thus we need to extend the notion of trace to solutions other than the fundamental one.

The author would like to thank the many mathematicians who contributed to his understanding of this material, and in particular Peter Gilkey for helpful discussions concerning differential operators on bundles.

\section{The heat equation on a homogeneous bundle}

Let $G$ be a compact, semisimple and simply connected Lie group which is simple modulo its center. Let $\pi_{\tau}$ be an irreducible representation on $G$ with highest weight $\tau$, so

$$
\pi_{\tau}: G \rightarrow \text { Aut } E \text {. }
$$

Most of the results still hold in the analogous situation when the representation is not irreducible. Let $\tilde{G}=G \times G$, and define the homogeneous vector bundle $\mathbf{E}_{\tau}$ by requiring that the following diagram commutes:

$$
\begin{array}{cccc}
\tilde{G} \times E & \rightarrow & \tilde{G} \times{ }_{\tau} E & =\mathbf{E}_{\tau} \\
\downarrow & & \downarrow \\
\tilde{G} & \rightarrow & \tilde{G} / \operatorname{diag} G \approx G .
\end{array}
$$

Here the bundle $\tilde{G} \times E$ is trivial and so has a Laplacian $\tilde{\Delta}$ on it defined by

$$
\tilde{\Delta}\left(\sum f_{i} e_{i}\right)=\sum\left(\tilde{\Delta} f_{i}\right) e_{i},
$$

where $\left\{e_{i}\right\}$ is a basis of $E$. Notice that (2.3) only holds because the bundle is trivial. The Laplacian on $\mathbf{E}_{\tau}$ is defined by

$$
(\Delta f)^{-}=\tilde{\Delta} \tilde{f}
$$


for $f$ a section of $\mathbf{E}_{\tau}, \tilde{f}$ the lifting of $f$ to a section of $\tilde{G} \times E$ and $(\Delta f)^{-}$the lifting of $\Delta f$. A section of $\tilde{G} \times E$ is called invariant if

$$
\tilde{f}(x \boldsymbol{\theta}, y \theta)=\pi_{\tau}(\theta) \tilde{g}(x, y) .
$$

There is a one-to-one correspondence between invariant sections of $\tilde{G} \times E$ and sections of $\mathbf{E}_{\tau}$. If we need to describe a section of $\mathbf{E}_{\tau}$ we shall usually do this by giving an invariant section of $\tilde{G} \times E$; this is the lifting of the section. To describe such a section as a function on $G$, rather than $\tilde{G}$, is done by using the splitting

$$
x \rightarrow(x, 1)
$$

of the last line of diagram (2.2). This splitting is not natural and so will only be used occasionally. Given any section $\tilde{f}$ of $\tilde{G} \times E$, not necessarily invariant, we can project it onto an invariant section $\tilde{f}_{\tau}$ by

$$
\tilde{f}_{\tau}(x, y)=\int_{G} \pi_{\tau}(\theta)^{-1} \tilde{f}(x \theta, y \theta) d \theta .
$$

Let $\langle$,$\rangle denote a G$-invariant inner product on $E,\{\lambda\}$ a complete set of eigenvalues, and $\left\{\phi_{\lambda}\right\}$ a complete orthonormal set of eigensections of $\mathbf{E}_{\tau}$. The fundamental solution of the heat equation on $\mathbf{E}_{\tau}$ is then

$$
H(x, y, t)=\sum \phi_{\lambda}(x) \phi_{\lambda}(y)^{*} e^{-\lambda t},
$$

which is regarded as an operator

$$
H(x, y, t) \nu(y)=\sum \phi_{\lambda}(x) \int\left\langle\phi_{\lambda}(y), \nu(y)\right\rangle d y e^{-\lambda t} .
$$

Notice that $H(x, y, t) \nu(y)$ does not depend on $y$; the variable $y$ is a dummy variable inserted to indicate the domains of the various functions. The function $H_{\nu}(x, t)=H(x, y, t) \nu(y)$ is the solution of the heat equation on $E_{\tau}$ with initial data $\nu(y)$. Taking $\nu(y)=\sum \phi_{\lambda}(y)$, which is a delta distribution, we obtain the fundamental solution of the heat equation as a section of $E_{\tau}$ :

$$
H(x, t)=\sum \phi_{\lambda}(x) e^{-\lambda t} ;
$$

and for $\nu(y)=\sum a_{\lambda} \phi_{\lambda}(y)$ we obtain the solution

$$
H_{a}(x, t)=\sum a_{\lambda} \phi_{\lambda}(x) e^{-\lambda t} .
$$

It is convenient to recover the operator form of the fundamental solution (2.8) from the section form (2.10). To do this we define the operator associated to the solution (2.11) to be

$$
H_{a}(x, y, t)^{*}=\sum a_{\lambda} \phi_{\lambda}(x) a_{\lambda} \phi_{\lambda}(y)^{*} e^{-\lambda t} .
$$

The trace of a solution is then the trace of the associated operator. This is

$$
\operatorname{tr} H_{a}^{*}(t)=\sum a_{\lambda}^{2} e^{-\lambda t},
$$


which in the case of the fundamental solution becomes

$$
Z(t)=\operatorname{tr} H^{*}(t)=\sum e^{-\lambda t} .
$$

Throughout this discussion each eigenvalue is repeated as often as its multiplicity.

\section{The heat equation on bundles over $S^{1}$}

We start by identifying the circle $S^{1}$ as $S^{1}=\{(\cos 2 \pi x, \sin 2 \pi x): x \in R / Z\}$ and taking a second copy: $S^{1}=\{(\cos 2 \pi y, \sin 2 \pi y)\}$. Then the product $\tilde{S}^{1}=S^{1}$ $\times S^{1}$ is formed. Let $\pi_{\tau}: S^{1} \rightarrow$ Aut $E_{\tau}$ be a representation of $S^{1}$ on a vector space $E_{\tau}$. We form the bundle $\mathbf{E}_{\tau}$ as in Diagram (2.2). The non-trivial irreducible representations of $S^{1}$ are, for each integer $k$, the two dimensional representations:

$$
\pi_{k}(\theta)=\left(\begin{array}{cc}
\cos 2 \pi k \theta & -\sin 2 \pi k \theta \\
\sin 2 \pi k \theta & \cos 2 \pi k \theta
\end{array}\right),
$$

where $E=\mathbf{C}^{2}$ and $\boldsymbol{\theta}=(\cos 2 \pi \theta, \sin 2 \pi \theta)$, with the group action $\mathbf{x}+\mathbf{y}=(\mathbf{x}+\mathbf{y})$. For the time being we shall only use irreducible $\pi_{k}$. A section of $\tilde{S}^{1} \times E$ is just a vector valued map $\tilde{f}: \tilde{S}^{1} \rightarrow E$. If this map is invariant, that is if $\tilde{f}(\mathbf{x}+\boldsymbol{\theta}$, $\mathbf{y}+\boldsymbol{\theta})=\pi_{k}(\boldsymbol{\theta}) \tilde{f}(\mathbf{x}, \mathbf{y})$, then $\tilde{f}$ defines a section of $\mathbf{E}_{k}$. The splitting (2.6) is now $x \rightarrow(x, 0)$.

Let $h_{1}(x, t)$ be the solution of the heat equation on functions on $S^{1}$. Then if

$$
\tilde{f}(x, y)=\left(\begin{array}{l}
f_{1}(x, y) \\
f_{2}(x, y)
\end{array}\right)
$$

is a section of $\tilde{S}^{1} \times E$ the section

$$
\left(\begin{array}{l}
\tilde{h}(x, y, t) * f_{1}(x, y) \\
\tilde{h}(x, y, t) * f_{2}(x, y)
\end{array}\right)
$$

is a solution of the heat equation. Here $\tilde{h}(x, y, t)=h_{1}(x, t) h_{1}(y, t)$, and * denotes convolution in the variables $x$ and $y$. We let

$$
\tilde{H}(x, y, t)=\left(\begin{array}{l}
\tilde{h}(x, y, t) \\
\tilde{h}(x, y, t)
\end{array}\right),
$$

and write (3.3) as $\tilde{H}(x, y, t) * \tilde{f}(x, y)$. A straightforward calculation shows that if $\tilde{f}$ is invariant, then

$$
\tilde{H}(x, y, t) * \tilde{f}(x, y)=\tilde{H}_{k}(x, y, t) * \tilde{f}(x, y) .
$$

We shall regard the section $\tilde{H}(x, 0, t)$ as the fundamental solution of the heat equation on the bundle $\mathbf{E}_{k}$ over $S^{1}$.

To calculate $\tilde{H}_{k}(x, 0, t)$ we first observe that

$$
h_{1}(x, t)=\sum_{n=0}^{\infty} \cos 2 \pi n x e^{-4 \pi^{2} n^{2} t} .
$$


Thus

$$
\tilde{H}(x, y, t)=\left(\begin{array}{l}
\sum \cos 2 \pi m x \cos 2 \pi n y e^{-4 \pi^{2}\left(n^{2}+m^{2}\right) t} \\
\sum \cos 2 \pi m x \cos 2 \pi n y e^{-4 \pi^{2}\left(n^{2}+m^{2}\right) t}
\end{array}\right)
$$

where both the sums are over all pairs of integers $m \geqslant 0, n \geqslant 0$. We now calculate

$$
\tilde{H}_{k}(x, y, t)=\int_{0}^{1} \pi_{k}(\theta)^{-1} \tilde{H}(x+\theta, y+\theta, t) d \theta,
$$

and find after an elementary, but lengthy calculation that

$$
\begin{aligned}
\tilde{H}_{\tau}(x, y, t)= & \frac{1}{4} \sum_{n=0}^{\infty}\left(\begin{array}{l}
(\cos 2 \pi(m x-(m-k) y)-\sin 2 \pi(m x-(m-k) y)) \\
(\cos 2 \pi(m x-(m-k) y)+\sin 2 \pi(m x-(m-k) y))
\end{array}\right) \\
& \cdot e^{-4 \pi^{2}\left(2 m^{2}-2 m k+k^{2}\right) t} \\
& +\frac{1}{4} \sum_{n=0}^{\infty}\left(\begin{array}{l}
(\cos 2 \pi(m x-(m+k) y)+\sin 2 \pi(m x-(m+k) y)) \\
(\cos 2 \pi(m x-(m+k) y)-\sin 2 \pi(m x-(m+k) y))
\end{array}\right) \\
& \cdot e^{-4 \pi^{2}\left(2 m^{2}+2 m k+k^{2}\right) t} .
\end{aligned}
$$

Setting $y=0$ yields the following expression for the fundamental solution of the heat equation $\mathbf{E}_{k}$ over $S^{1}$ :

$$
\begin{aligned}
\tilde{H}(x, 0, t)= & \frac{1}{2} \sum_{m=0}^{\infty}\left(\begin{array}{l}
\cos 2 \pi m x \cosh 8 \pi^{2} m k t-\sin 2 \pi m x \sinh 8 \pi^{2} m k t \\
\cos 2 \pi m x \cosh 8 \pi^{2} m k t+\sin 2 \pi m x \sinh 8 \pi^{2} m k t
\end{array}\right) \\
& \cdot e^{-4 \pi^{2}\left(2 m^{2}+k^{2}\right) t} .
\end{aligned}
$$

We further observe that we can carry out an analogous calculation starting with other initial data. We replace the solution $h_{1}$ in equation (3.6) by

$$
h(x, t)=\sum_{n=0}^{\infty} a_{n} \cos 2 \pi n x e^{-4 \pi^{2} n^{2} t},
$$

where $a_{n}$ are just constants giving initial data $\nu$. The corresponding solution $H_{v}(x, 0, t)$ on $\mathbf{E}_{\tau}$ over $S^{1}$ is then:

$$
\begin{aligned}
& H_{\nu}(x, 0, t)=\frac{1}{4} \sum_{m=0}^{\infty} a_{m} e^{-4 \pi^{2}\left(2 m^{2}+k^{2}\right) t} \\
& \cdot\left\{a_{m+k}\left(\begin{array}{l}
\cos 2 \pi m x-\sin 2 \pi m x \\
\cos 2 \pi m x+\sin 2 \pi m x
\end{array}\right) e^{8 \pi^{2} k m t}\right. \\
& \left.+a_{m-k}\left(\begin{array}{l}
\cos 2 \pi m x+\sin 2 \pi m x \\
\cos 2 \pi m x-\sin 2 \pi m x
\end{array}\right) e^{-8 \pi^{2} k m t}\right\} .
\end{aligned}
$$

Here we have extended the notation so that when $r$ is negative the constant $a_{r}=a_{-r}$. We let $H_{\nu}$ be the resulting solution on $\mathbf{E}_{k}$ of $h$. 


\section{Reduction to the maximal torus}

We start with vector valued functions

$$
\tilde{f}: \tilde{G} \rightarrow E .
$$

If $\tilde{f}$ is invariant under conjugation, then

$$
\bar{j} \bar{f} \mid \tilde{T}=\Delta_{T} \tilde{j} \tilde{f}-4 \pi^{2}\|\tilde{\rho}\|^{2} \tilde{j} \tilde{f},
$$

where

$$
\tilde{j}(x, y)=j(x) j(y)
$$

with $j$ the usual denominator function on $T: j(x)=\prod_{\alpha>0} 2 i \sin 2 \pi \alpha(x)$. Notice that our conventions have introduced a factor $2 \pi$ with each root and a factor $4 \pi^{2}$ with $\|\rho\|^{2}$. The other notation is that $\tilde{\Delta}$ is the Laplacian on $\tilde{G}, \tilde{\Delta}_{T}$ is the flat Laplacian on $\tilde{T}$, and $\tilde{\rho}$ is half the sum of the positive roots of $\tilde{G}$, so that $\|\tilde{\rho}\|^{2}=2\|\rho\|^{2}$. This result is due to Harish-Chandra and its use is discussed in [2]. Using equation (4.2) we make the following definition.

Definition 4.1. If $\tilde{H}(x, y, t)$ is a solution of the heat equation on $\tilde{G}$, then the associated solution is $\tilde{H}_{T}(x, y, t)=e^{8 \pi^{2}\|\rho\|^{2}} \tilde{j}(x, y) \tilde{H}(x, y, t)$ for $(x, y) \in \tilde{T}$.

THEOREM 4.2. The associated solution is a solution of the flat heat equation on $\tilde{T}$, $\tilde{\Delta}_{T} u-\partial u / \partial t=0$.

Proof. This is essentially the same as in [1], the only difference being that we are working on $\tilde{G}$ rather than $G$.

We denote with abuse of notation, the initial data of $\tilde{H}(x, y, t)$ by $\tilde{H}(x, y, 0)$. Then we wish to relate the initial data of $\tilde{H}$ with that of $\tilde{H}_{T}$, the associated solution. In terms of Fourier series the result is as follows.

THEOREM 4.3. If $\tilde{H}(x, y, 0)=\sum a_{\lambda_{\mu}} \chi_{\lambda}(x) \chi_{\mu}(y)$ then $\tilde{H}_{T}(x, y, 0)=$ $\sum b_{\alpha \beta} e^{2 \pi i(\alpha(x)+\beta(y))}$ where

$$
b_{\alpha \beta}=\left\{\begin{array}{cl}
a_{\lambda \mu}(-1)^{\sigma \tau} & \text { for } \lambda=\sigma^{-1} \alpha-\rho, \mu=\tau^{-1} \beta-\rho, \\
0 & \text { if either } \alpha \text { or } \beta \text { is singular. }
\end{array}\right.
$$

Here the first sum is over $\lambda, \mu$ dominant weights and the second is over all weights $\alpha$, $\beta$.

REMARK. If both $\alpha$ and $\beta$ are regular, that is if neither is in a wall of the Weyl diagram, then there are unique $\sigma$ and $\tau$ such that $\sigma \alpha-\rho$ and $\tau \beta-\rho$ are dominant. Points $\gamma$ in the walls have the property that $\omega \gamma=\gamma$ for some $\omega$ in the Weyl group with $(-1)^{\omega}=-1$, in which case $\sigma \gamma-\rho$ is never dominant. 
Proof. This result is a straightforward calculation. The Weyl character formula

$$
\chi_{\lambda}(x)=\sum_{\alpha \in W}(-1)^{\alpha} e^{2 \pi i \sigma(\lambda+\rho) x} / j(x)
$$

is substituted into the expression for $\tilde{H}(x, y, 0)$. Rearranging the sums then gives the result. Note that $a_{\lambda_{\mu}}$ and $b_{\alpha \beta}$ are both elements of the vector space $E$.

THEOREM 4.4. If $H(\tilde{x}, t)$ is the fundamental solution on the trivial bundle $\tilde{G} \times E$ over $\tilde{G}$ then $H_{\tilde{T} \tau}=H_{r}$.

Proof. Let $e_{1}, \ldots, e_{m}$ be an orthonormal basis for $E$. Then

$$
H(\tilde{x}, t)=h(\tilde{x}, t) \sum e_{i},
$$

where $h(\tilde{x}, t)$ is the fundamental solution of the heat equation for functions on $\tilde{G}$. Equation (4.5) just states that on a trivial bundle we can work component by component. Now we calculate

$$
H_{\tilde{T}_{\tau}}=e^{-8 \pi^{2}\|\rho\|^{2} t} \int_{\operatorname{diag} T} \tilde{j}(x+\theta) h(\tilde{x} \theta, t) \pi_{\tau}(\theta)^{-1} \sum e_{i} d \theta,
$$

and

$$
H_{\tau \tilde{T}}=e^{-8 \pi^{2}\|\rho\|^{2} \tilde{j}} \tilde{j}(\tilde{x}) \int_{\operatorname{diag} G} h(x+\theta, t) \pi_{\tau}(\theta)^{-1} \sum e_{i} d \theta .
$$

In both of these equations the action of $\theta$ is understood to be multiplication by $(\theta, \theta)$ in the diagonal subgroup of either $\tilde{G}$ or $\tilde{T}$. Observe that both $H_{\tilde{T}_{\tau}}$ and $H_{\tau \tilde{T}}$ satisfy the flat heat equation on $\tilde{T}$ and from (4.6) and (4.7) both have

$$
\nu(\tilde{x})= \begin{cases}0, & \tilde{x} \notin \operatorname{diag} T, \\ \tilde{j}(\tilde{x}) \pi_{\tau}(x) \sum e_{i}, & \tilde{x} \in \operatorname{diag} T,\end{cases}
$$

as initial data. Since these sections have the same initial data and satisfy the same equation they are equal. This completes the proof.

\section{The trace of a solution of the heat equation}

We are interested in the case of the fundamental solution. To compute this we shall pass to the associated solution on the maximal torus $T$, which is different from the fundamental solution on $T$. So we interpret (2.13) independently of eigensection expansions. The trace of the solution is

$$
\operatorname{tr} H_{a}(t)=\int_{G}\left\langle H_{a}(x, t), H_{a}(x, 0)\right\rangle d x
$$


We now consider the associated solution on the maximal torus $\tilde{T}$ of $\tilde{G}$ :

$$
\tilde{H}_{T}(x, y, t)=e^{8 \pi^{2}\|\rho\|^{2}} \tilde{j}(x, y) \tilde{H}(x, y, t) .
$$

Let $\tilde{Z}_{T}(t)=\operatorname{tr} \tilde{H}_{T}(t)$ and $\tilde{Z}(t)=\operatorname{tr} H(t)$ be the traces of the associated solution and the solution, respectively, and let $|W|$ denote the order of the Weyl group.

LEMMA 5.1. The traces are related by

$$
\tilde{Z}_{T}(t)=e^{8 \pi^{2}\|\rho\|^{2}} \tilde{Z}(t) /|W|
$$

Proof. Calculate $\tilde{Z}_{T}$ and use the Weyl integration formula.

We note that the trace $\tilde{Z}$ on $\tilde{G}$ is related to the trace $Z$ on $G$.

LEMMA 5.2. The traces are related by

$$
\tilde{Z}(t)=\operatorname{vol}(G) Z(t) .
$$

Proof. This is a straightforward calculation:

$$
\begin{aligned}
\tilde{Z}(t) & =\iint_{\tilde{G}}\langle\tilde{H}(x, y, t), \tilde{H}(x, y, 0)\rangle d x d y \\
& =\iint_{\tilde{G}}\left\langle\tau(y)^{-1} \tilde{H}\left(x y^{-1}, 1, t\right) \tau(y)^{-1} \tilde{H}\left(x y^{-1}, 1,0\right)\right\rangle d x d y \\
& =\iint_{\tilde{G}}\langle\tilde{H}(x, 1, t), \tilde{H}(x, 1,0)\rangle d x d y \\
& =\operatorname{vol} G \int_{G}\langle\tilde{H}(x, 1, t), \tilde{H}(x, 1,0)\rangle d x .
\end{aligned}
$$

The step to equation (5.4) uses the invariance of the solution, (5.5) uses the invariance of both the inner product and Haar measure. The final equation (5.6) is the result in the lemma.

We now calculate the trace of a solution of the heat equation on $S^{1}$. Let

$$
h(x, t)=\sum a_{n} \cos (2 \pi n x) e^{-4 \pi^{2} n^{2} t}
$$

be a solution of the heat equation for functions on $S^{1}$ giving rise to the solution $H_{\nu}(x, 0, t)$ of equations (3.12). The trace of $H_{\nu}$ is then given by the following result.

LEMMA 5.3. The trace of $H_{v}$ is

$$
Z_{\nu}(t)=\frac{1}{8} \sum a_{m}^{2}\left(a_{m+k}^{2} e^{8 \pi^{2} m k t}+a_{m-k}^{2} e^{-8 \pi^{2} m k t}\right) e^{-4 \pi^{2}\left(2 m^{2}+k^{2}\right) t} .
$$


Proof. This is a straightforward calculation with equation (3.12) substituted into equation (5.1).

The situation on the maximal torus $T$ is analogous. Let $\alpha$ be a weight of the representation $\tau$ which defines the bundle $E_{r}$. Then $\alpha$ is a two-dimensional representation of $T$ :

$$
\alpha(x)=\left(\begin{array}{rr}
\cos 2 \pi \alpha(x) & -\sin 2 \pi \alpha(x) \\
\sin 2 \pi \alpha(x) & \cos 2 \pi \alpha(x)
\end{array}\right) .
$$

The solution for functions over $T$ becomes $h(x, t)=\sum \alpha_{\lambda} \cos 2 \pi \lambda(x) e^{-4 \pi^{2}\|\lambda\|^{2} t}$ where the sum is over $\lambda \in P$, the lattice of weights of $G$. The resulting trace is then given in the next lemma.

LEMMA 5.4. The trace of the resulting solution in $E_{\alpha}$ is

$$
Z_{\alpha}(t)=\frac{1}{8} a_{\lambda}^{2}\left(a_{\lambda+\alpha}^{2} e^{8 \pi^{2}\langle\lambda, \alpha\rangle t}+a_{\lambda-\alpha}^{2} e^{-8 \pi^{2}\langle\lambda, \alpha\rangle t}\right) e^{-4 \pi^{2}\left(2\|\lambda\|^{2}+\|\alpha\|^{2}\right) t} .
$$

Proof. This lemma is proved in the same way as the previous lemma. The main changes in the calculation are that $m$ is replace by $\lambda, k$ by $\alpha, m^{2}$ by $\|\lambda\|^{2}, k^{2}$ by $\|\alpha\|^{2}$, and $m k$ by the inner product $\langle\lambda, \alpha\rangle$.

To calculate the trace of the fundamental solution of the heat equation on $E_{\tau}$ we need to identify the coefficients $a_{\lambda}$. First consider the case of the solution for functions, that is $\tau=0$. Define the polynomial $d$ by

$$
d(\lambda)=\prod_{\alpha>0}\langle\lambda, \alpha\rangle /\langle\rho, \alpha\rangle
$$

then the fundamental solution of the heat equation is

$$
H(x, t)=\sum_{\lambda \in P \cap D} d(\lambda+\rho) \chi_{\lambda}(x) e^{-\|\lambda+\rho\|^{2}+\|\rho\|^{2} t} .
$$

The associated solution on $T$ is $H_{T}(x, t)=j(x) e^{-\|\rho\|^{2} t} H(x, t)$; that is, using the Weyl character formula we have

$$
H_{T}(x, t)=\sum_{\lambda \in P \cap D} d(\lambda+\rho)\left(\sum_{\sigma \in W}(-1)^{\sigma} e^{2 \pi i \sigma(\lambda+\rho)(x)}\right) e^{-\|\lambda+\rho\|^{2} t} .
$$

As in [1] we can use the skew invariance of $d$ under the Weyl group to write this as

$$
H_{T}(x, t)=\sum_{\lambda \in P} d(\lambda) e^{2 \pi i \lambda(x)} e^{-\|\lambda\|^{2} t},
$$

where the shift by $\rho$ has been removed. If we start with the solution

$$
H(x, t)=\sum a_{\lambda} \cos 2 \pi \lambda(x) e^{-\|\lambda\|^{2} t},
$$


and if we form the product solution $h(x, t) h(y, t)$ on $\tilde{T}$ and make this invariant, then the resulting solution on $T$ is

$$
h_{\tau}(x, t)=\sum \frac{a_{\lambda}^{2} e^{2 \pi i \lambda(x)}}{2} e^{-\|\lambda\|^{2} t} .
$$

Thus the associated fundamental solution is the resulting solution with the choice of coefficients

$$
a_{\lambda}=\sqrt{2 d(\lambda)} .
$$

Returning to the case when $\tau$ is no longer trivial we observe that the same result still holds.

LEMMA 5.5. Let $a_{\lambda}=\sqrt{2 d(\lambda)}$. Then the resulting solution of $\sum a_{\lambda} e^{2 \pi i \lambda(x)} e^{-\|\lambda\|^{2} t}$ is the associated fundamental solution.

Proof. Since both the resulting solution and the associated solution satisfy the heat equation, it is sufficient to check that they have the same initial data. The initial data is supported at the identity element of the group, and so we can work locally. Since the bundle $\mathbf{E}_{\tau}$ is locally trivial, the result now follows from the result for functions.

The trace of the fundamental solution can now be calculated.

THEOREM 5.6. Let $Z(t)$ be the trace of the fundamental solution of the heat equation on the bundle $E_{\tau}$ over $G$. Then

$$
Z(t)=\frac{e^{-8 \pi^{2}\|\rho\|^{2} t}}{|W|} \sum m_{\tau}(\alpha) d(\lambda) d(\lambda+\alpha) e^{-4 \pi^{2}\left(\|\lambda\|^{2}+\|\lambda+\alpha\|^{2}\right) t},
$$

where $m_{\tau}(\alpha)$ is the multiplicity of weight $\alpha$ in the representation $\tau$ and the sum is over all weights $\alpha$ of $\tau$ and all weights $\lambda$.

Proof. By Lemma 5.1, we have

$$
\tilde{Z}(t)=e^{-8 \pi^{2}\|\rho\|^{2} t} \tilde{Z}_{T}(t) /|W|
$$

Now the bundle $E_{\tau}$ over $T$ decomposes as a direct sum

$$
\mathbf{E}_{\tau} \mid T=\oplus \mathbf{E}_{\alpha}
$$

over all the weights $\alpha$. Thus

$$
\tilde{Z}_{T}(t)=\sum m_{\tau}(\alpha) Z_{\alpha}(t)
$$

and the result follows by substituting

$$
a_{\lambda}^{2}=2 d(\lambda)
$$

in Lemma 5.4 and noticing that the multiplicity $m_{\tau}(\alpha)$ is the same as $m_{\tau}(-\alpha)$, so that there is cancellation when we sum over $\alpha$. 


\section{The Clebsch-Gordan numbers}

By the natural extension of the result in [2] we can calculate the eigenvalues of $\Delta$ and their multiplicities.

Lemma 6.1. (a) The eigenvalues of the Laplacian on $\mathbf{E}_{\tau}$ are $4 \pi^{2}(c(\lambda)+c(\mu))$ where $c(\lambda)=\|\lambda+\rho\|^{2}-\|\rho\|^{2}$ and $\mu$ is the highest weight of an irreducible representation in the decomposition

$$
\pi_{\lambda} \otimes \pi_{\tau}^{*}=\sum m_{\lambda \tau^{*}}(\mu) \pi_{\mu} .
$$

(b) The multiplicity of this eigenvalue is $m_{\lambda r^{*}}(\mu)\left(\operatorname{dim} \pi_{\mu}\right)^{2}$.

Now $\operatorname{dim} \pi_{\mu}=d(\mu+\rho)$, and so substituting into (2.14) gives

$$
Z(t)=e^{-8 \pi^{2}\|\rho\|^{2} t} \sum_{\lambda, \mu} m_{\lambda \tau^{*}}(\mu) d(\mu+\rho)^{2} e^{-4 \pi^{2}\left(\|\lambda+\rho\|^{2}+\|\mu+\rho\|^{2}\right) t} .
$$

If we set $\alpha=\lambda-\mu$ in Theorem 5.6 and equate coefficients, we obtain the formula

$$
\sum m_{\lambda \tau^{*}}(\mu) d(\mu+\rho)^{2}=\frac{1}{|W|} \sum m_{\tau}(\lambda-\mu) d(\lambda+\rho) d(\mu+\rho),
$$

where in both sums $\|\lambda+\rho\|^{2}+\|\mu+\rho\|^{2}=n$, a fixed constant, and where, in the first sum, $\lambda$ and $\mu$ are dominant weights, but in the second they are just weights.

\section{References}

[1] H. D. Fegan, 'The heat equation on a compact Lie group', Trans. Amer. Math. Soc. 246 (1978), 339-357.

[2] H. D. Fegan, 'The spectrum of the Laplacian on forms over a Lie group', Pacific J. Math. 90 (1980), 373-387.

[3] H. D. Fegan, 'Differential equations on Lie groups and tori, the wave equation and Huygen's Principle', Rocky Mountain J. Math. 14 (1984), 699-704.

[4] Howard D. Fegan and Peter B. Gilkey, 'Invariants of the heat equation', Pacific J. Math. 117 (1985), 233-254.

[5] V. K. Patodi, 'Curvature and the eigenforms of the Laplace operator', J. Differential Geom. 5 (1971), 233-249.

[6] N. R. Wallach, Harmonic analysis on homogeneous spaces (M. Dekker, New York, 1973).

Department of Mathematics and Statistics

University of New Mexico

Albuquerque, New Mexico 87131

U.S.A. 